\title{
CONGLOMERADO DE CASOS DE TUBERCULOSIS MULTIDROGORRESISTENTE EN UN COLEGIO DEL DISTRITO DE ICA, PERÚ
}

\author{
Julio Torres ${ }^{1, a}$, Victoria Sardón²,b Mirtha G. Soto ${ }^{3, b}$, Rolado Anicama ${ }^{4, b}$, \\ C. Hugo Arroyo-Hernández ${ }^{5, b}$, César V. Munayco ${ }^{3, c}$
}

\begin{abstract}
RESUMEN
Se describe la evolución y las características de los casos de un conglomerado de tuberculosis multidrogorresistente (MDR) ocurrido el año 2001, en un centro educativo localizado en una zona urbano marginal del distrito de Ica, Perú. Se identificó 15 escolares que estuvieron relacionados entre ellos antes de enfermar de tuberculosis. El promedio de edad fue 15 años. Doce casos fueron MDR y siete fueron resistentes a las cinco drogas de primera línea (RHEZS), cinco de los casos recibieron tres diferentes esquemas de tratamiento antituberculoso; el tiempo promedio de tratamiento antituberculoso fue de 37 meses (mínimo 21 y máximo 59 meses). Trece casos curaron y dos fallecieron. El presente estudio documenta un conglomerado de casos de TB-MDR en un centro educativo que, por los vínculos epidemiológicos y la simultaneidad en que aparecieron, podría tratarse de un probable brote de TB-MDR, con un desenlace satisfactorio luego de un tratamiento prolongado.
\end{abstract}

Palabras clave: Tuberculosis; Tuberculosis resistente a múltiples medicamentos; Brotes de enfermedades; Escuelas; Niños; Perú (fuente: DeCS BIREME).

\section{CLUSTER OF MULTIDRUG-RESISTANT TUBERCULOSIS CASES IN A SCHOOL OF THE DISTRICT OF ICA, PERU}

\begin{abstract}
We describe the evolution and features of a cluster of Multidrug-resistant tuberculosis (MDR TB) cases that occurred in 2001, in a school located in a sub-urban area of the district of Ica, Peru. We identified 15 students related before becoming infected with tuberculosis. The mean age of the cluster was 15 years. A total of 12 students were MDR-TB cases and 7 were drug-resistant to 5 first-line drugs (RHEZS). Five out of the 15 cases received at least 3 different anti-tuberculosis treatment schemes. The average treatment duration was 37 months (minimum 21 and maximum 59 months). A total of 13 cases recovered and 2 died. This study describes a cluster of MDR -TB cases in an educational facility, which due to the epidemiological link and time presentation, is probably an outbreak of MDR TB with a satisfactory outcome after prolonged treatment.
\end{abstract}

Key words: Tuberculosis; Multidrug-resistant tuberculosis; Disease outbreaks; Schools; Children; Peru (source: MeSH NLM).

\section{INTRODUCCIÓN}

La tuberculosis (TB) continúa siendo un problema de salud pública mundial que afecta principalmente a países en vías de desarrollo. En la última década, las estrategias de control se han visto afectadas por la emergencia de la coinfección con el VIH y la farmacorresistencia (TB-MDR y XDR), y la débil respuesta de los servicios de salud ${ }^{(1,2)}$. La tuberculosis representa el $2,5 \%$ de la carga global de enfermedad y se ubica como la sexta causa de muerte a nivel mundial ${ }^{(2,3)}$.
Desde inicios del tercer milenio nuestro país afronta un incremento en los casos de TB MDR, los informes epidemiológicos oficiales sobre TB de nuestro país, comparado con otros, muestran que Perú es el sétimo país a nivel mundial con mayor problemática de TB-MDR y cuarto en número absoluto estimado de casos ${ }^{(2)}$.

Los brotes o conglomerados de tuberculosis tienen mayor probabilidad de presentarse en poblaciones vulnerables como son: personas privadas de libertad, poblaciones indígenas, refugiados, en colegios, etc.; que por sus

\footnotetext{
Hospital Regional Docente de Ica. Ica, Perú.

2 Centro de Salud. Sr. de Luren, DIRESA Ica. Ica, Perú

3 Grupo Temático de Tuberculosis y Lepra. Dirección General de Epidemiología del Ministerio de Salud. Ica, Perú.

4 Oficina de Epidemiología. Dirección Regional de Salud de Ica.

5 Facultad de Medicina, Universidad Nacional San Luis Gonzaga. Ica, Perú.

a Médico Neumólogo; b Médico Cirujano; ' Médico Epidemiólogo
}

Recibido: 07-04-11 Aprobado: 24-08-11 
características ecológicas (contacto permanente y prolongado, hacinamiento, pobreza, abandono social, entre otros factores) facilitan la transmisión de tuberculosis o su reactivación ${ }^{(4,5)}$.

Dentro de estas poblaciones se encuentran los escolares, principalmente de centros educativos localizados en zonas de alta transmisión de tuberculosis, donde la exposición a sintomáticos respiratorios con tuberculosis (sensible, MDR o XDR) no identificados o pacientes en inicio de tratamiento (familiares, compañeros de estudio, profesores, etc.), incrementan el riesgo de infección y tienen más probabilidad de desarrollar enfermedad tuberculosa activa que otras poblaciones ${ }^{(6)}$.

En octubre de 2001, en el Hospital Regional Docente de la Provincia de Ica se hospitalizaron dos adolescentes, uno con diagnóstico de tuberculosis pulmonar con baciloscopía positiva y el otro con tuberculosis enteroperitoneal con baciloscopía negativa. Dos a tres semanas después ambos casos se discutieron en juntas médicas, debido a la mala evolución y falta de respuesta al tratamiento antituberculoso con esquemas primarios.

En la junta médica del segundo caso se estableció la relación de que ambos pacientes estudiaban en el mismo centro educativo de un pueblo joven del distrito de Ica. Lo que originó una investigación epidemiológica cuyo objetivo fue identificar la presencia de más casos en dicho colegio y tomar medidas de prevención y control.

Durante esta investigación se identificaron nueve escolares más que recibían tratamiento antituberculoso en diferentes establecimientos de salud y, la mayoría, eran de una misma aula. Además, se realizó una búsqueda activa de sintomáticos respiratorios en todos los estudiantes, profesores y trabajadores administrativos del centro educativo, detectándose en el mes de noviembre dos casos más.

El presente estudio describe el conglomerado de casos de TB-MDR ocurrido en un colegio secundario y el desenlace clínico de los casos.

\section{EL ESTUDIO}

Se realizó un estudio clínico epidemiológico de todos los casos de tuberculosis procedentes de un centro educativo de un área urbano-marginal del distrito de lca, ubicado en el departamento de Ica, en la costa Sur del Perú, entre 2001 y 2009; donde, se detectó en 2001 un conglomerado de casos de tuberculosis.
Para ello se elaboró una ficha clínico-epidemiológica en la que se recopiló información de los expedientes del comité de evaluación de retratamientos intermedios (CERI) de la DIRESA Ica y de los registros de los establecimientos de salud; estos expedientes contienen datos de historia clínica, fichas de seguimiento y evaluación, hoja de tratamiento, resultados de cultivo y perfil de sensibilidad de cada uno de los casos con tuberculosis identificados. Además, se realizó un estudio de contactos de los casos con tuberculosis, de acuerdo con la normativa de la Estrategia Sanitaria Nacional de Prevención y Control de Tuberculosis ${ }^{(1)}$.

Para identificar a los casos se les asignó un número correlativo del 1 al 15 , de acuerdo con la fecha de su primer diagnóstico de tuberculosis.

Los resultados de las pruebas de sensibilidad fueron recogidas de las historias clínicas de los pacientes, estas pruebas se realizaron en el Instituto Nacional de Salud (INS). Las muestras de cuatro casos que fracasaron entre 2001 y 2003 fueron procesadas en el laboratorio del Instituto de Massachuset de la Universidad de Harvard, Estados Unidos, porque en ese momento el INS no realizada pruebas de sensibilidad para drogas de segunda línea.

\section{HALLAZGOS}

Entre 2001 y 2009 se identificaron quince escolares con tuberculosis, procedentes del mismo centro educativo, siete fueron mujeres, con una edad promedio al momento del diagnóstico de 15 años (mínimo de 13 y un máximo de 19 años).

\section{DESCRIPCIÓN DE LOS CASOS}

De total de casos identificados, solo el caso uno (probablemente el que inició el conglomerado de casos) tenía antecedente de enfermedad tuberculosa previa; que fue diagnosticada por primera vez en 1992, recibió tratamiento Esquema I y egresó como curado; en 2000 volvió a presentar enfermedad tuberculosa y recibió tratamiento Esquema II, pero fracasó en el tercer mes de tratamiento, por lo que fue diagnosticado como TB-MDR por su perfil de resistencia. Al momento que se identificó el conglomerado, en octubre de 2001, este caso ya se encontraba en su tercer mes de tratamiento para TB-MDR.

Once casos fueron diagnosticados entre marzo y noviembre de 2001 y, por ser casos nuevos, iniciaron tratamiento con esquemas primarios (Esquema I, III), pero cinco de estos casos fracasaron dos a tres meses después de iniciado su tratamiento y fueron 
diagnosticados como TB-MDR. Otros cinco casos egresaron como curados después de haber recibido esquema primario completo, pero meses después (entre 4 y 36 meses) volvieron a ser diagnosticados de tuberculosis e iniciaron tratamiento para TB-MDR porque eran parte del conglomerado de casos. Solo un caso se curó con esquema primario y no volvió a ser diagnosticado de tuberculosis al menos durante el periodo de estudio.

Tres casos fueron diagnosticados de tuberculosis entre 2004 y 2006 y, por el nexo ya conocido con los casos anteriores, iniciaron directamente tratamientos para TB-MDR.

\section{TRATAMIENTO Y CONDICIÓN DE EGRESO DE LOS CASOS}

En total doce casos recibieron esquemas primarios como primer tratamiento, estos esquemas tenían una duración de seis a nueve meses e incluían cinco drogas de primera línea (rifampicina, isoniacida, etambutol, pirazinamida y estreptomicina). Todos los casos, excepto uno, volvieron a presentar enfermedad tuberculosa activa.

Ocho casos recibieron esquema estandarizado como segundo tratamiento, este esquema, en aquel entonces, tenía una duración de 18 meses e incluía seis a siete drogas: dos drogas de primera línea (pirazinamida y etambutol) y cuatro a cinco drogas de segunda línea (kanamicina, capreomicina, etionamida, cicloserina y ácido aminosalicílico). De estos casos solo uno curó con este esquema, dos fallecieron y cinco fracasaron.

Once casos recibieron esquemas de "tratamiento individualizado" (estos esquemas se estructuraban según el perfil de sensibilidad de cada paciente y tenían una duración aproximada de 24 meses), tres de los cuales recibieron este esquema como primer tratamiento, otros tres como su segundo tratamiento y cinco como su tercer tratamiento.

El promedio de tiempo total que estuvieron en tratamiento (ya sea esquema primario o secundario) los quince casos fue de 41 meses (mínimo de tres y máximo de 95 meses) y el promedio de tiempo que estuvieron en tratamiento para TB-MDR (solo esquema estandarizado y esquemas individualizados) fue de 37 meses con un mínimo de 21 y máximo de 59 meses.

Con respecto a la condición de egreso, del total de casos trece curaron y dos fallecieron (Figura 1).

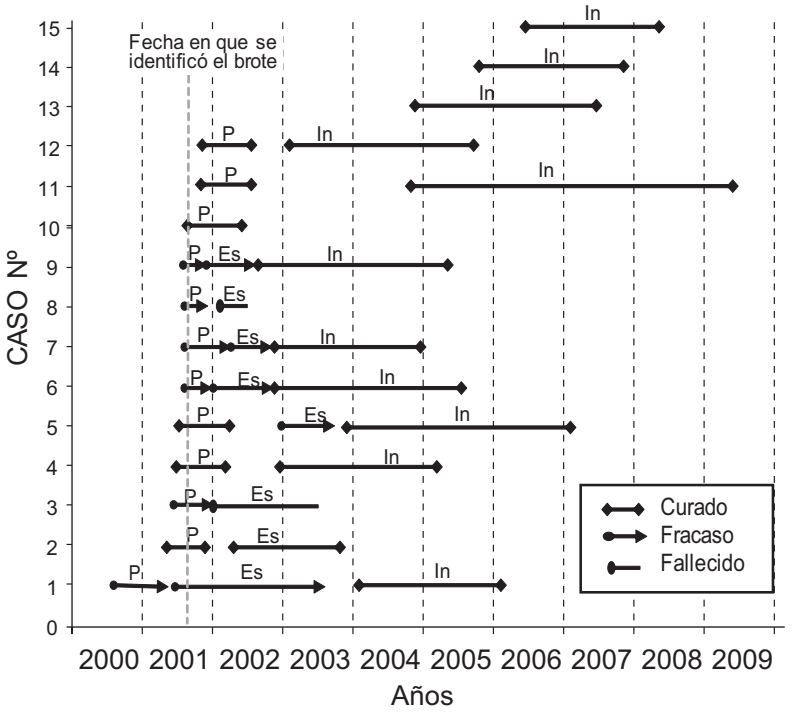

Figura 1. Evolución y Tratamientos recibidos por los pacientes con TB-MDR en un colegio secundario de Ica.

$\mathrm{P}$ : tratamiento primario (esquema I, II, III), Es: esquema de retratamientos estandarizado, In: esquema de retratamiento Individualizado.

\section{ESTUDIO DE CONTACTOS}

Del conglomerado identificado, trece casos estuvieron relacionados porque estudiaron en la misma aula el 2001; además, los casos dos y cinco tenían vínculos sentimentales mientras que los casos once y doce, tenían vínculos amicales estrechos además de ser vecinos. Por otro lado, el caso ocho y el caso cuatro no pertenecían a la misma aula que los casos anteriores, pero el caso ocho era familiar cercano del caso seis, que pertenecía al conglomerado.

El caso cuatro no tenía ninguna relación familiar o amical conocida con alguno de los casos anteriores, pero tenía el antecedente de contacto con un tío con tuberculosis sensible, que curó con el tratamiento recibido. Por otro lado, el caso cinco tenía el antecedente de contacto con su hermano con tuberculosis sensible, el cual curó con tratamiento.

El caso uno tenía un hermano mayor (con el cual vivía y compartía la misma habitación), que fue diagnosticado por primera vez con tuberculosis el 2000 y que fracasó cuatro meses después de iniciado su tratamiento con Esquema I, por lo que paso a recibir Esquema II, egresó como curado después de completar el tratamiento, pero cuatro meses después recayó e inicio esquema de retratamiento estandarizado para TB-MDR, al término del tratamiento fue dado de alta como curado. Es importante notar que estos dos casos presentaron TBMDR casi simultáneamente (Figura 2). 


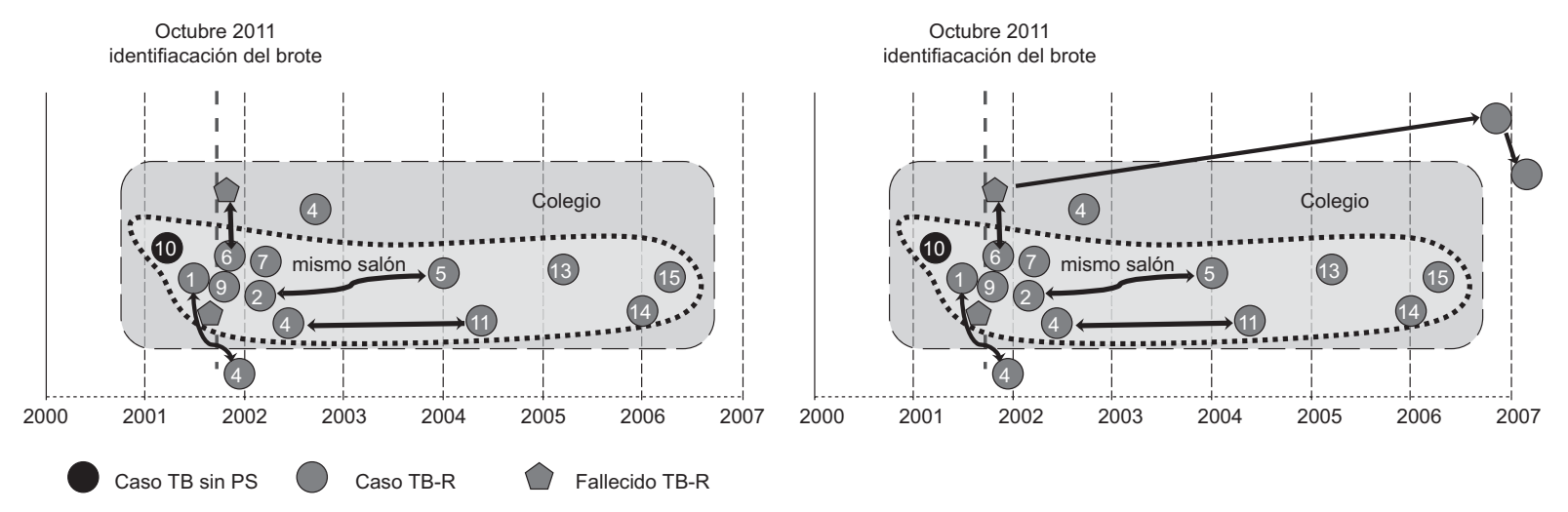

Figura 2. Estudio de contactos de casos con TB-MDR en un colegio secundario de Ica 2001-2006.

*La numeración indica el orden cronológico del primer diagnóstico de los casos; la posición indica el orden de ingreso a tratamientos con esquemas secundarios (estandarizado o Individualizado); las flechas continuas indican vínculos amicales, familiares u otros.

\section{PERFIL DE SENSIBILIDAD}

Se logró conseguir el perfil de sensibilidad de doce de los quince casos. De los tres restantes, a un caso no se le realizó perfil de sensibilidad porque curó con tratamiento primario y, de los otros dos no se pudo tener acceso a los resultados de su perfil de sensibilidad porque fueron trasferidos a otra región.

El patrón de resistencia para drogas de primera línea fue variable, pero todos fueron MDR (resistentes $\mathrm{RH}$ ), siete casos fueron resistentes a las cinco drogas de primera línea; tres casos fueron resistentes a cuatro drogas (RHEZ o RHZS) y dos casos fueron resistentes a tres drogas $(\mathrm{RHZ})$.

Seis de siete casos, que tuvieron perfil de sensibilidad para drogas de segunda línea, fueron resistentes a etionamida y solo un caso fue resistente, además, a capreomicina, kanamicina y amikacina, todos los demás casos fueron sensibles a estas drogas (Figura 3).

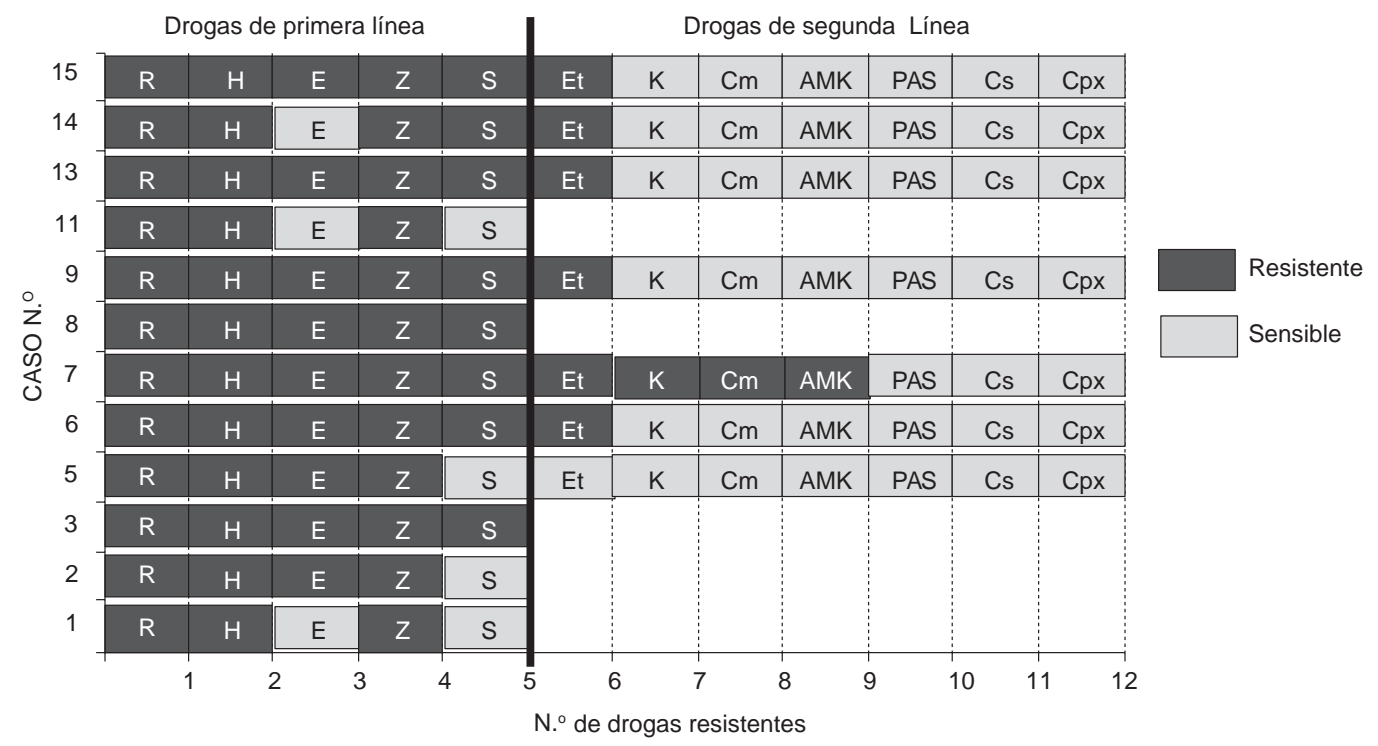

Figura 3. Perfil de Sensibilidad de casos con TB-MDR en un colegio secundario de Ica 2001-2006.

$\mathrm{R}$ : rifampicina, H: isoniacida, E: etambutol, Z: pirainamida, S: estreptomicina, Et: etionamida, K:kanamicina, Cm: capreomicina, AMK: amikacina, PAS, ácido aminosalicilico, Cs: cicloserina, Cpx: ciprofloxacino. 


\section{DISCUSIÓN}

Se identificó un conglomerado de casos de TB-MDR entre los alumnos de un colegio localizado en una zona urbano-marginal ubicada al noreste del distrito de Ica, lográndose determinar que estos casos estuvieron relacionados entre ellos antes de enfermar de tuberculosis.

Si bien existe un vínculo epidemiológico entre los casos del conglomerado, no se podría afirmar que es un brote debido a que no se realizó el estudio genético de las muestras de los casos, que confirme que las cepas estuvieron genéticamente relacionadas entre si ${ }^{(5)}$.

Todos los pacientes que fueron diagnosticados entre 2001 y 2003, recibieron al menos dos esquemas, y más de la mitad recibieron tres esquemas de tratamiento con un prologado tiempo de tratamiento. Esto se debió a la evolución de las políticas terapéuticas para TB-MDR en nuestro país, que se inició en 1997 con el esquema estandarizado y que dos años más tarde implementó el esquema individualizado ${ }^{(3)}$.

En Ica, el primer caso que recibió tratamiento para TBMDR fue en $1998^{\left({ }^{8}\right)}$. El ingreso al esquema estandarizado en aquel momento, estaba dirigido solo a pacientes que fracasaban o recaían dos e incluso tres veces a esquemas primarios, el esquema individualizado estaba indicado solo para los que fracasaban a esquema estandarizado. Recién a partir de 2005, se norma la atención de los pacientes con TB-MDR en el país, estableciéndose lineamientos para los esquemas de tratamiento y la duración de estos ${ }^{(7)}$.

De acuerdo con la norma actual (9), estos pacientes hubieran sido considerados como contacto de paciente MDR y hubieran ingresado directamente a esquemas para TBMDR, como sucedió con los dos últimos casos de este conglomerado (detectados en 2006), que recibieron un esquema individualizado con un menor tiempo de tratamiento. Por lo que resaltamos la importante del antecedente de contacto para la decisión terapéutica inicial.

El conocer los patrones de resistencia a fármacos antituberculosos es crucial para el éxito en el tratamiento de los pacientes (7,10); desafortunadamente, en el momento que se detectó este conglomerado, se tenía una capacidad limitada para determinar los patrones de resistencia ${ }^{(7)}$ y los perfiles de sensibilidad eran realizados en función de la evolución clínica, bacteriológica y según la respuesta a los tratamientos recibidos. Por lo que en la mayoría de los casos de este conglomerado no se determinó el perfil de sensibilidad antes del inicio del primer tratamiento, impidiendo conocer el patrón inicial de resistencia.
Probablemente el patrón de resistencia de los casos de este conglomerado hayan sido influenciados por los tratamientos recibidos, sin embargo, todos los casos con prueba de sensibilidad fueron MDR, aunque tuvieron diferentes patrones de resistencia.

Los centros educativos son instituciones con mayor vulnerabilidad a tener brotes de enfermedades infectocontagiosas de trasmisión aérea, debido a la alta tasa de contacto y al prolongado tiempo de exposición que existe en esta población (11,12). En los centros educativos ubicados en zonas de pobreza y con alta trasmisión de tuberculosis se incrementa el riesgo de infectarse y progresar a enfermedad activa, debido a que es más frecuente la exposición a sintomáticos respiratorios con tuberculosis no identificados o pacientes en la primera etapa de su tratamiento, ya sea en su núcleo familiar, su entorno social o en el mismo colegio.

En países desarrollados, donde la tuberculosis no es endémica, se han descrito brotes de tuberculosis en escolares, los cuales se iniciaron por el contacto con familiares ${ }^{(13,14)}$, o conductores de autobuses escolares ${ }^{(15)}$, en estos casos la investigación de los brotes se basó en la identificación de tuberculosis latente de los contactos a través de la prueba de PPD.

En países en vías de desarrollo como el nuestro, donde la tuberculosis es hiperendémica, la identificación de un brote de tuberculosis se debe realizar a través de estudios genéticos del $M$. Tuberculosis (RFLP, spoligotyping) (6), ya que la prevalencia de infección por este bacilo es alta ${ }^{(7)}$.

El presente estudio describe un conglomerado de tuberculosis MDR en un centro educativo, de un área de alta trasmisión de tuberculosis que, por los vínculos epidemiológicos y la simultaneidad en que aparecieron, podría tratarse de un probable brote de TB-MDR, que pone en evidencia la necesidad de implementar un sistema de vigilancia epidemiológica en centros educativos y otras instituciones cerradas, como asilos, centros penitenciarios, albergues, entre otros.

\section{Contribuciones de autoría}

Todos los autores participaron en la concepción y diseño del estudio. JT, VS, MS, RA y HA participaron en la recolección de datos. JT, MS y CM participaron en el análisis e interpretación de datos, MS y CM participaron en la redacción del manuscrito. Todos los autores participaron en la revisión crítica del manuscrito y aprobaron su versión final.

\section{Fuentes de financiamiento}

Autofinanciado. 


\section{Descargo de responsabilidad}

Los contenidos de este manuscrito solamente son responsabilidad de los autores y no necesariamente representan a opinión oficial de las instituciones a las que pertenecen.

\section{REFERENCIAS BIBLIOGRÁFICAS}

1. Chiang CY, Centis R, Migliori GB. Drug-resistant tuberculosis: past, present, future. Respirology. 2010;15(3):413-32.

2. Organización Panamericana de la Salud. Tuberculosis en las Américas. Reporte 2009 [Internet]. Washington, DC: OPS/OMS; 2009 [citado el 13 de febrero de 2011]. Disponible en: http://new.paho.org/hq/index.php?option=com_cont ent\&task=view\&id=2701\&ltemid=394\&lang=es

3. Ministerio de Salud del Perú, Dirección General de Salud de las Personas. Construyendo Alianzas Estratégicas para detener la tuberculosis: Experiencia Peruana. Lima: MINSA; 2006

4. Gomez DR, Diaz RR undefined García AN, Valdivia AJ. Estudio epidemiológico-molecular de un brote de tuberculosis en el hospital psiquiátrico de la habana. Rev Cubana Hig Epidemiol. 2000;38(3):201-9.

5. Karuchit S, Diem L, Rienthong D, Tappero JW, ljaz K, Maloney SA, et al. Multidrug-resistant tuberculosis outbreak among US-bound Hmong refugees, Thailand, 2005. Emerging Infect. Dis. 2008;14(11):1715-21.

6. Singh V, Kaur S. Multi-drug resistant childhood tuberculosis. Indian J Pediatr. 2011;78(4):456-63.

7. García PE y Rodriguez JC. Epidemiología molecular de la tuberculosis: principales hallazgos y su aplicación en España. Arch Bronconeumol. 2005;41(11):618-24.
8. Torres Chang J. Tuberculosis resistente en la provincia de Ica: situación actual, factores asociados y evaluación de esquemas terapéuticos. MINSA 1998 - 2004. Lima: MINSA; 2005.

9. Ministerio de Salud del Perú. Norma técnica de salud para el control de la Tuberculosis. 1a ed. Lima: FIMART; 2006.

10. Vijayasekaran D. Treatment of childhood tuberculosis. Indian J Pediatr. 2011;78(4):443-8.

11. Yang Y, Sugimoto JD, Halloran ME, Basta NE, Chao DL, Matrajt L, et al. The transmissibility and control of pandemic influenza A (H1N1) virus. Science. 2009;326(5953):729-33.

12. Dye $\mathbf{C}$, Williams BG. The population dynamics and control of tuberculosis. Science. 2010;328(5980):856-61.

13. Sacks JJ, Brenner ER, Breeden DC, Anders HM, Parker RL. Epidemiology of a tuberculosis outbreak in a South Carolina junior high school. Am J Public Health. 1985;75(4):361-5.

14. Phillips L, Carlile J, Smith D. Epidemiology of a tuberculosis outbreak in a rural Missouri high school. Pediatrics. 2004;113(6):e514-9.

15. Yusuf HR, Braden CR, Greenberg AJ, Weltman AC, Onorato IM, Valway SE. Tuberculosis transmission among five school bus drivers and students in two New York counties. Pediatrics. 1997;100(3):E9.

Correspondencia: César Munayco Escate,

Dirección General de Epidemiologia; Calle Daniel Olaechea 199. Jesús María. Lima 11. Lima, Perú.

Teléfono: 511-4614307

Correo electrónico: cmunayco@dge.gob.pe

\section{Consulte la versión electrónica de la Revista Peruana de Medicina Experimental y Salud Pública en}

wwW.scopus.com
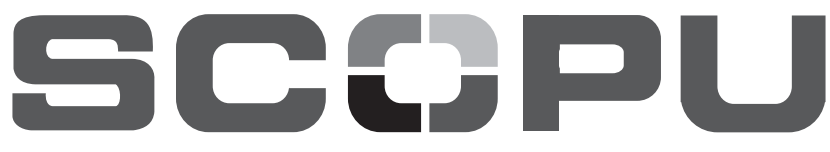

TM

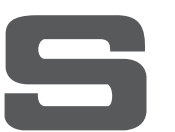

